\title{
Efeitos cardiovasculares da medetomidina e cetamina em Puma concolor e tempo de recuperação após aplicação de ioimbina ou atipamezole
}

\author{
[Cardiovascular effects of medetomidine and cetamine in Puma concolor and recovery time after \\ application of ioimbin or atipamezole]
}

\author{
S.M. Caramalac ${ }^{1}$, A.R. Oliveira ${ }^{2}$, V.B. Albuquerque ${ }^{3}$, T. Deco-Souza ${ }^{4}$, F.O. Frazílio ${ }^{4 *}$ \\ ${ }^{1}$ Aluno de pós-graduação - Faculdade de Medicina Veterinária e Zootecnia - Universidade \\ Federal de Mato Grosso do Sul - Campo Grande, MS \\ ${ }^{2}$ Aluno de pós-graduação - Faculdade de Medicina Veterinária e Zootecnia - Universidade \\ Estadual Paulista - Botucatu, SP \\ ${ }^{3}$ Médica veterinária autônoma - Campo Grande, MS \\ ${ }^{4}$ Faculdade de Medicina Veterinária e Zootecnia - Universidade Federal de Mato \\ Grosso do Sul - Campo Grande, MS
}

\begin{abstract}
RESUMO
O objetivo deste estudo foi avaliar as alterações cardiorrespiratórias causadas pela medetomidina associada à cetamina, e o tempo de recuperação após aplicação intramuscular de atipamezole ou ioimbina em Puma concolor. Para isso, foi realizada a aplicação de medetomidina $(100 \mu \mathrm{g} / \mathrm{kg})$ associada à cetamina $(5 \mathrm{mg} / \mathrm{kg})$ em 11 onçaspardas, sendo os parâmetros cardiorrespiratórios registrados a cada 15 minutos, durante 90 minutos de avaliação. Em seguida, a anestesia foi revertida com aplicação intramuscular de ioimbina $(0,4 \mathrm{mg} / \mathrm{kg} ; \mathrm{n}=5)$ ou atipamezole $(0,25 \mathrm{mg} / \mathrm{kg} ; \mathrm{n}=6)$, sendo analisado o tempo até a recuperação. Dos parâmetros cardiorrespiratórios avaliados, houve diferença apenas na frequência respiratória (entre os momentos 60 e 90 minutos), estando esta, todavia, dentro do intervalo de referência para a espécie. Além disso, verificou-se tempo para decúbito esternal significativamente menor nos animais do grupo atipamezole (18 \pm 7 minutos), quando comparado ao grupo ioimbina ( $36 \pm 17$ minutos), entretanto o tempo de recuperação completa foi estatisticamente igual entre os dois reversores analisados. Assim, a associação anestésica promoveu anestesia eficiente, segura e de rápida indução em onças-pardas, permitindo a imobilização dos animais durante os 90 minutos de avaliação, sem a ocorrência de complicações. Ao se comparar a reversão anestésica com atipamezole e ioimbina, observou-se equivalência dos fármacos no tempo de recuperação completa dos animais.
\end{abstract}

Palavras-chave: agonistas $\alpha$-2 adrenérgicos, animais silvestres, tranquilização

\begin{abstract}
The aim of this study was to evaluate the cardiorespiratory changes caused by ketamine-associated medetomidine, and the recovery time after intramuscular application of atipamezole or yohimbine in Puma concolor. For this, the application of medetomidine $(100 \mu \mathrm{g} / \mathrm{kg})$ associated with ketamine $(5 \mathrm{mg} / \mathrm{kg})$ was performed in eleven brown ounces, and the cardiorespiratory parameters were recorded every 15 minutes during 90 minutes of evaluation. Afterwards, anesthesia was reversed with intramuscular application of yohimbine $(0.4 \mathrm{mg} / \mathrm{kg} ; \mathrm{n}=5)$ or atipamezole $(0.25 \mathrm{mg} / \mathrm{kg} ; n=6)$, and time to recovery was analyzed. Of the cardiorespiratory parameters evaluated, there was a difference only in respiratory rate (between 60 and 90 minutes), however, within the reference range for the species. In addition, there was a significantly shorter time for sternal decubitus

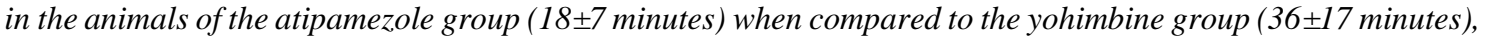
however the complete recovery time was statistically equal between the two reversers analyzed. Thus, the anesthetic association promoted efficient, safe and fast induction anesthesia in puma, allowing the animals to be immobilized during the 90 minutes of evaluation without complications. Comparing anesthetic reversal with atipamezole and yohimbine, drug equivalence was observed in the complete recovery time of the animals.
\end{abstract}

Keywords: $\alpha-2$ adrenergic agonists, wild animals, tranquilization

Recebido em 10 de setembro de 2018

Aceito em 16 de março de 2020

*Autor para correspondência (corresponding author)

E-mail: fabricio.frazilio@ufms.br 


\section{INTRODUÇÃO}

O manuseio de grandes felídeos é altamente arriscado à equipe envolvida; assim, em consequência de sua natureza selvagem, é necessária a imobilização química desses animais para realização de manejo clínico seguro (Goodrich et al., 2001; Lescano et al., 2014). Contudo, dados na literatura sobre os efeitos dos anestésicos utilizados para imobilizar essas espécies ainda são escassos (Lescano et al., 2014; Albuquerque et al., 2016). Algumas combinações farmacológicas já foram utilizadas para a imobilização de onças-pardas, e consideradas efetivas, como associações tiletamina-zolazepam, cetamina e xilazina (Lescano et al., 2014) ou detomidina e cetamina (Albuquerque et al., 2016), por exemplo. Entretanto, a disponibilidade comercial, o tempo de duração da imobilização, o preço e a segurança do produto são fatores que interferem na escolha do anestésico.

Entre as opções disponíveis no mercado, a medetomidina é um sedativo da classe dos agonistas $\alpha-2$ adrenérgicos pouco estudado em felinos selvagens. Ela é considerada um sedativo mais potente devido à sua afinidade 10 vezes maior pelos receptores $\alpha-2$ adrenérgicos, quando comparada à xilazina (Langan et al., 2000), o que torna sua utilização preferida para a anestesia de carnívoros. Apesar de também estar relacionada ao desenvolvimento de bradicardia e bloqueios atrioventriculares, como os demais sedativos da mesma classe, a medetomidina produz intensa sedação (Raszplewiscz et al., 2013; Cardoso et al., 2014; Canfrán et al., 2016), e a sua combinação com cetamina permite uma anestesia geral para a realização de procedimentos simples e associados a bloqueios locorregionais, bem como para a realização de pequenas cirurgias em animais domésticos e não domésticos (Miller et al., 2003), como drenagem de abscessos, suturas, biópsias e retirada de nódulos cutâneos, por exemplo.

Pela impossibilidade de realização de avaliação clínica pré-anestésica em animais selvagens, é importante que reversores estejam à disposição da equipe, para tratamento de eventuais efeitos adversos ou aprofundamento inadequado da anestesia. Além disso, é fundamental que se conheça em quanto tempo após a aplicação do reversor os animais restabelecem seu comportamento normal, tanto para a segurança deles no ambiente como para as pessoas envolvidas na captura. Os reversores para os $\alpha-2$ agonistas atualmente disponíveis no mercado são o atipamezole e a ioimbina. A principal diferença entre os dois reversores é a sua seletividade e afinidade aos receptores, nos quais o atipamezole tem maior seletividade $\alpha-2 / \alpha-1$ do que a ioimbina, ao passo que a afinidade de ambos os reversores é semelhante para os receptores de subtipos $\alpha-2 \mathrm{~A}$, $\alpha-2 B$ e $\alpha-2 C$, enquanto para os receptores $\alpha-2 D$, a ioimbina possui pouca especificidade em baixas doses (Haapalinna et al., 1997). Pouco se sabe sobre as diferenças de respostas aos antagonistas $\alpha-2$ na espécie aqui estudada.

Dessa forma, os objetivos do presente estudo foram avaliar os possíveis efeitos cardiovasculares da medetomidina associada à cetamina em onças-pardas (Puma concolor), além do tempo de recuperação desses animais após a reversão dos seus efeitos com atipamezole ou ioimbina.

\section{MATERIAL E MÉTODOS}

Os procedimentos foram realizados nas dependências do Centro de Reabilitação de Animais Silvestres (Cras) de Campo Grande, Mato Grosso do Sul. Foram anestesiadas 11 onças-pardas, para a realização de exames de rotina (hemograma, bioquímicos e ultrassonografia abdominal). Para isso, foram submetidos a jejum alimentar prévio de 12 horas, sem jejum hídrico, e permaneceram em seu recinto de origem, a fim de se evitar estresse e manipulação excessiva.

Devido à natureza selvagem dos animais, os dados anestésicos analisados não puderam ser aferidos no momento basal (antes da aplicação do protocolo anestésico). O peso foi estimado de acordo com o escore corporal, com o auxílio do registro no prontuário de contenções anteriores. Para a anestesia, foi utilizada medetomidina $(100 \mu \mathrm{g} / \mathrm{kg})$ (Precision Pharmacy, CA, USA) e $5 \mathrm{mg} / \mathrm{kg}$ de cetamina (Vetaset, Fort Dodge Saúde Animal, Campinas, SP, Brasil), associadas no mesmo dardo aplicado por via intramuscular (IM), na região do músculo semimembranoso, por meio de zarabatana.

Após a aplicação do dardo, o animal foi observado e avaliado conforme descrito por Lescano et al. (2014), para registro do tempo até o primeiro 


\section{Caramalac et al.}

efeito (sinais de sonolência, ataxia e queda da cabeça), tempo até decúbito e tempo de indução (avaliado pela ausência de resposta a estímulos na cabeça e membros torácicos com cambão). Associadamente, a profundidade da anestesia foi avaliada por meio da utilização da escala de avaliação numérica (Tab. 1). Em casos em que o animal não apresentasse escore 4 de anestesia após 30 minutos (min) da aplicação do dardo, uma dose complementar com metade da dose inicial foi instituída por via intramuscular.

Tabela 1. Escala de avaliação numérica para pontuação de sedação, adaptada de Porters et al. (2014)

\begin{tabular}{ll}
\hline Escore & \multicolumn{1}{c}{ Definição } \\
\hline 0 & Completamente acordado, capaz de levantar e andar, postura normal. \\
1 & Levanta, mas cambaleia quando tenta andar. \\
2 & $\begin{array}{l}\text { Decúbito esternal, capaz de levantar a cabeça, ocasionalmente faz fracas e frustradas } \\
\text { tentativas de levantar. }\end{array}$ \\
3 & $\begin{array}{l}\text { Decúbito lateral, levemente responsivo ao bater de palmas e à manipulação (pode levantar } \\
\text { ligeiramente a cabeça, a cauda ou o membro). }\end{array}$ \\
\hline
\end{tabular}

Ao apresentarem escore de sedação 4, as onças foram retiradas do recinto, pesadas por meio de balança suspensa - Pesola, colocadas sobre mesa cirúrgica em decúbito lateral direito e paramentadas (Fig. 1) para aferição da frequência cardíaca (FC), frequência respiratória (FR), temperatura retal (TR), saturação de oxihemoglobina por oxímetro de pulso posicionado na língua e pressão arterial média (PAM), sistólica (PAS) e diastólica (PAD) pelo método oscilométrico, por meio de monitor multiparamétrico (DX 2010, Dixtal, Manaus, AM, Brasil). Os animais foram entubados com sonda traqueal de tamanho 9,5 com ventilação espontânea de ar ambiente, para coleta de dados da capnografia. (Fig. 2).
Um cateter intravenoso (20 G Insyte; BD, Juiz de Fora, MG, Brasil) foi introduzido na veia cefálica direita, para fluidoterapia, com solução de ringer com lactato, em taxa de manutenção $(5 \mathrm{~mL} / \mathrm{kg} / \mathrm{h})$. Para monitoração da pressão arterial sistólica, diastólica e média pelo método oscilométrico, um manguito com largura correspondente a $40 \%$ do diâmetro da região radioulnar foi colocado nesse respectivo local. Em casos de redução do escore de sedação, com demonstração de sinais de despertar (movimentos voluntários de membros, cauda e cabeça), preconizou-se administrar bolus de propofol (Propovan, Cristália, São Paulo, SP, Brasil) dose-efeito.

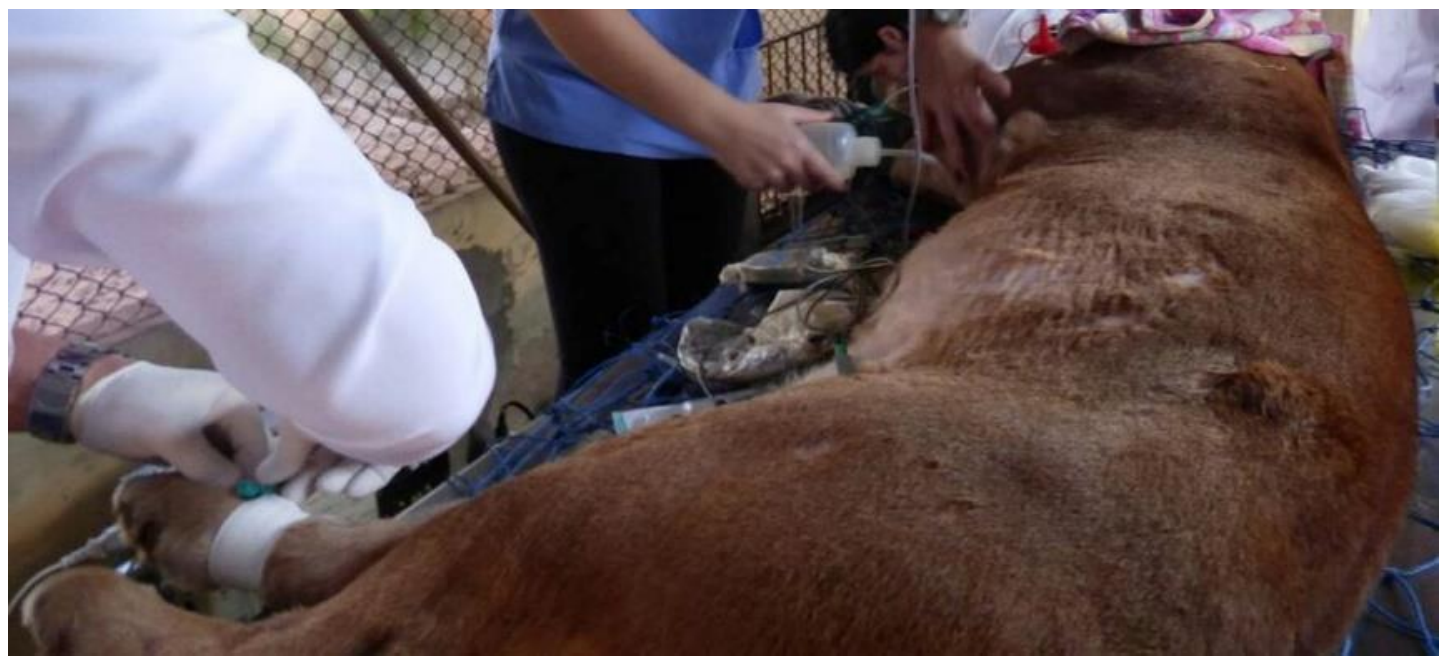

Figura 1. Puma concolor sendo paramentada com eletrodos de eletrocardiograma, para monitoração anestésica. 


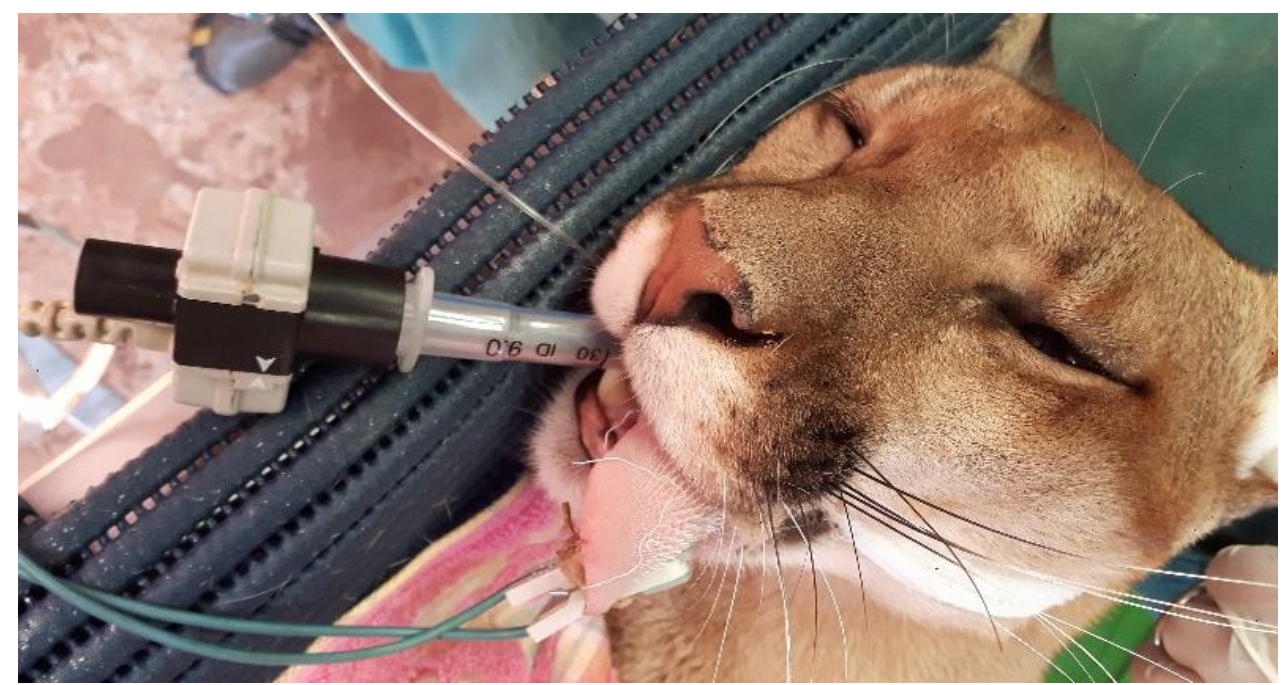

Figura 2. Puma concolor entubada com sonda orotraqueal, para monitoração capnográfica, respirando ar ambiente. $\mathrm{O}$ oxímetro está colocado na língua, para monitoração de oximetria e pulso.

Os pacientes tiveram os parâmetros cardiorrespiratórios registrados a cada $15 \mathrm{~min}$ (a partir do momento em que eram retirados do recinto), totalizando $90 \mathrm{~min}$ de avaliação (momento 15min [M15]; momento 30min [M30]; momento 45min [M45]; momento 60min [M60]; momento 75min [M75]; momento 90min [90]). Após o período de avaliação, os animais foram realocados em seus respectivos recintos, onde foram distribuídos aleatoriamente em dois grupos, para aplicação intramuscular dos reversores atipamezole (Antisedan, Zoetis, Brasil), na dose de $0,25 \mathrm{mg} / \mathrm{kg}$ (grupo atipamezole [GA]; $\mathrm{n}=6$ ), ou ioimbina (Ioimbina 1\%, Nutrivet Farmácia Veterinária, Jaguariúna, São Paulo, Brasil), na dose de $0,4 \mathrm{mg} / \mathrm{kg}$ (grupo ioimbina [GI]; $\mathrm{n}=5$ ).

Todas as onças foram observadas pelos mesmos dois avaliadores, logo após a aplicação do reversor, os quais não sabiam a que grupo os animais pertenciam, correspondendo a uma avaliação do tipo cego. Durante a recuperação, foi avaliado o tempo necessário até o animal ficar em posição esternal (tempo para decúbito esternal [TEst]) e o tempo para locomoção sem ataxia (tempo para recuperação [TRecup]).

Os dados foram analisados por estatística descritiva para comparação entre médias e medianas, intervalos de confiança e distribuição de frequências (histograma) para suposição de normalidade. Todos os dados considerados como paramétricos para as amostras dependentes foram submetidos à análise de variância (ANOVA twoway), sendo as médias comparadas por meio do teste de Tukey, considerando-se nível de significância de $5 \% \quad(\mathrm{P}<0,05)$. Os dados não paramétricos em amostras independentes foram avaliados por Mann-Withney.

\section{RESULTADOS}

A média e desvio-padrão de peso dos animais foi $45 \pm 15,2 \mathrm{~kg}$, sendo, assim, utilizados $100 \pm 10 \mu \mathrm{g} / \mathrm{kg}$ de medetomidina associada a $4,8 \pm 0,6 \mathrm{mg} / \mathrm{kg}$ de cetamina. Após aplicação do anestésico, o tempo transcorrido para a observação dos primeiros efeitos (como cabeça baixa ou ataxia) foi de $4 \pm 2$ min. Passados $8 \pm 5$ min após a aplicação, os animais entravam em decúbito esternal e, em $13 \pm 10 \mathrm{~min}$, os animais eram considerados anestesiados, período no qual era possível sua manipulação.

Das 11 onças anestesiadas, seis receberam dose ligeiramente inferior à preconizada, três receberam a dose previamente estipulada e duas receberam dose ligeiramente acima do preconizado (Tab. 2). Dos animais que receberam a dose ligeiramente inferior, em quatro foi necessária a reaplicação de um segundo dardo devido à imobilização insatisfatória. Daqueles que receberam a dose adequada, foi necessária a reaplicação do dardo em apenas um animal. 


\section{Caramalac et al.}

Tabela 2. Dose utilizada de medetomidina, cetamina e propofol (os dois últimos em $\mathrm{mg} / \mathrm{kg}$ ) para contenção química de cada animal da espécie Puma concolor

\begin{tabular}{lccc} 
Medetomidina & $\begin{array}{c}\text { Cetamina } \\
(\mu \mathrm{g} / \mathrm{kg})\end{array}$ & $\begin{array}{c}\text { Propofol } \\
(\mathrm{mg} / \mathrm{kg})\end{array}$ \\
\hline Onça 1* & 136,35 & 6,81 & - \\
Onça 2 & 104,16 & 5,2 & - \\
Onça 3* & 109,99 & 5,04 & - \\
Onça 4 & 100 & 5 & 3,62 \\
Onça 5 & 88,8 & 4,4 & 0,88 \\
Onça 6* & 150 & 7,5 & - \\
Onça 7* & 138,9 & 6,9 & 1,48 \\
Onça 8* & 139,99 & 6,9 & - \\
Onça 9 & 97,22 & 4,9 & 6,11 \\
Onça 10 & 116,6 & 5,8 & 6,66 \\
Onça 11 & 100 & 5 & 3,33 \\
Média & $116,6 \pm 21,07$ & $5,77 \pm 1.06$ & - \\
\hline
\end{tabular}

*Animais nos quais foram necessárias reaplicações da metade da dose inicial de medetomidina e cetamina.

Apesar da diferença significativa entre os momentos M60 e M90 no parâmetro FR (Tab. 3), os valores mantiveram-se dentro do esperado para a espécie. Não foram observadas alterações no padrão respiratório dos animais. Os demais parâmetros apresentaram-se estáveis, sem diferenças estatísticas entre os momentos e os grupos avaliados. As principais arritmias cardíacas observadas foram as contrações ventriculares prematuras (CVP), que ocorreram com frequência média de $4 \pm 2$, em nove das 11 onças anestesiadas, durante toda a monitoração anestésica. Bloqueios atrioventriculares (BAV) ocorreram apenas em um animal (animal 10), havendo dois BAV de primeiro grau e um BAV de segundo grau no período de avaliação. O tempo para decúbito esternal e o tempo para recuperação das onças-pardas (em minutos) estão descritos na Tab. 4. Foram observadas diferenças $(\mathrm{P}=0,04)$ entre os grupos GA e GI para o tempo decorrido para o animal ficar em posição esternal, entretanto não foram observadas diferenças $(\mathrm{P}>0,05)$ entre os grupos quanto ao tempo total de recuperação.

Tabela 3. Média e desvio-padrão de frequência cardíaca (FC, em batimentos por minuto), pressão arterial sistólica (PAS, em mmHg), pressão arterial média (PAM, em mmHg), pressão arterial diastólica (PAD, em $\mathrm{mmHg}$ ), frequência respiratória (FR, em movimentos torácicos por minuto), saturação de oxi-hemoglobina $\left(\mathrm{SpO}_{2}\right.$, em \%), dióxido de carbono ao final da expiração $\left(\mathrm{EtCO}_{2}\right)$ e temperatura retal $\left(\mathrm{TR}\right.$, em $\left.{ }^{\circ} \mathrm{C}\right)$ de 11 onças (Puma concolor) anestesiadas com medetomidina e cetamina, durante 90 minutos de monitorização

\begin{tabular}{|c|c|c|c|c|c|c|c|}
\hline \multirow{2}{*}{ Parâmetros } & \multicolumn{6}{|c|}{ Momentos } & \multirow{2}{*}{ P-value } \\
\hline & M15 & M30 & M45 & M60 & M75 & M90 & \\
\hline FC & $90 \pm 14$ & $88 \pm 11$ & $87 \pm 13$ & $86 \pm 13$ & $87 \pm 14$ & $88 \pm 12$ & $\mathrm{P}=0,28$ \\
\hline PAS & $134 \pm 34$ & $136 \pm 33$ & $137 \pm 35$ & $133 \pm 32$ & $138 \pm 26$ & $136 \pm 26$ & $\mathrm{P}=0,98$ \\
\hline PAM & $118 \pm 29$ & $120 \pm 28$ & $118 \pm 26$ & $112 \pm 22$ & $119 \pm 18$ & $115 \pm 17$ & $\mathrm{P}=0,87$ \\
\hline PAD & $109 \pm 27$ & $114 \pm 26$ & $108 \pm 24$ & $102 \pm 30$ & $111 \pm 18$ & $101 \pm 14$ & $\mathrm{P}=0,51$ \\
\hline FR & $24 \pm 11$ & $27 \pm 15$ & $27 \pm 11$ & $29 \pm 13 *$ & $24 \pm 8$ & $23 \pm 5^{*}$ & $\mathrm{P}=0,01$ \\
\hline $\mathrm{SpO}_{2}$ & $94 \pm 4$ & $94 \pm 3$ & $96 \pm 2$ & $96 \pm 1$ & $96 \pm 2$ & $96 \pm 1$ & $\mathrm{P}=0,17$ \\
\hline $\mathrm{EtCO}_{2}$ & $32 \pm 4$ & $32 \pm 3$ & $32 \pm 3$ & $32 \pm 4$ & $32 \pm 3$ & $33 \pm 3$ & $\mathrm{P}=0,55$ \\
\hline TR & $38,3 \pm 1,6$ & $38,0 \pm 1,6$ & $38,2 \pm 1,6$ & $38,0 \pm 1,8$ & $37,9 \pm 1,7$ & $38 \pm 1,5$ & $\mathrm{P}=0,12$ \\
\hline
\end{tabular}

*P<0,05 diferença entre os momentos.

Tabela 4. Média e desvio-padrão do tempo (minutos) para decúbito esternal (TEst) e do tempo para recuperação (TRecup) das 11 onças-pardas (Puma concolor) anestesiadas com medetomidina-cetamina, cuja reversão foi realizada com atipamezole (GA) ou ioimbina (GI).

\begin{tabular}{ccc} 
& TEst & TRecup \\
\hline GA & $18 \pm 7^{*}$ & $63 \pm 23$ \\
GI & $36 \pm 17^{*}$ & $92 \pm 24$ \\
\hline
\end{tabular}

$* \mathrm{P}<0,05$ diferença estatística significativa entre os momentos. 


\section{DISCUSSÃO}

Não foram encontrados trabalhos publicados que descrevam quais são os valores hemodinâmicos e respiratórios em onças-pardas anestesiadas com a associação farmacológica utilizada. Todavia, a utilização de medetomidina $(60-80 \mu \mathrm{g} / \mathrm{kg})$ com cetamina $(2,5-3 \mathrm{mg} / \mathrm{kg})$ propiciou completa imobilização de leopardo-das-neves, além de estabilidade nos parâmetros cardiovasculares, mantendo-se entre 84 e 112bpm (Jalanka, 1989a). Em animais da espécie Puma concolor, a associação de detomidina $(0,15 \mathrm{mg} / \mathrm{kg})$ e cetamina $(5 \mathrm{mg} / \mathrm{kg})$ resultou em parâmetros cardiorrespiratórios semelhantes aos obtidos no presente estudo (Albuquerque et al., 2016). Estabilidade cardiovascular também foi observada por Tomizawa et al. (1997) e Rockhill et al. (2011), que utilizaram anestesia dissociativa para a contenção química de felídeos silvestres.

Entretanto, a manutenção dos parâmetros hemodinâmicos após contenção química com anestésicos dissociativos não foi observada em outros estudos (Lamgan et al., 2000; Jacquier et al., 2006; Sontakke et al., 2009; Johansson et al., 2013; Lescano et al., 2014). Valores superiores de frequência cardíaca em onças-pardas, após a imobilização com tiletamina-zolazepam $(2 \mathrm{mg} / \mathrm{kg})$, cetamina $(1,6 \mathrm{mg} / \mathrm{kg})$ e xilazina $(0,4 \mathrm{mg} / \mathrm{kg})$, podem ser atribuídos à menor seletividade dos fármacos utilizados, levando à menor redução dos batimentos cardíacos (Lescano et al., 2014). Esse efeito cronotrópico negativo causado pelos agonistas $\alpha-2$ adrenégicos altamente seletivos se evidencia nos achados de Johansson et al. (2013), que, ao utilizarem medetomidina $(0,01 \pm 0,04 \mathrm{mg} / \mathrm{kg})$ com tiletaminazolazepan $(2,17 \pm 0,45 \mathrm{mg} / \mathrm{kg})$ em leopardos-dasneves, observaram redução significativa das frequências cardíaca e respiratória, valores semelhantes aos obtidos no presente estudo. Esses achados podem ser atribuídos à atividade nos receptores $\alpha-2$ adrenérgicos pré-sinápticos, levando à inibição da liberação de noradrenalina, com consequente bradicardia (Virtanen et al., 1988).

As principais arritmias cardíacas observadas com o uso de medetomidina associada à cetamina foram a contração ventricular prematura e o bloqueio atrioventricular, ambas com baixa frequência de ocorrência e atribuídas aos efeitos diretos do agonista alfa-2 adrenérgico no sistema nervoso autônomo (Alves et al., 2000).Tipicamente, essas arritmias não são ameaçadoras à vida (Sinclair, 2003). As arritmias ventriculares requerem intervenção médica quando geram sintomas, são contínuas ou produzem disfunção ventricular esquerda (Yamada, 2016).

Houve relativa estabilidade na frequência respiratória, com oscilação significativa apenas em dois momentos (aumento no M60 e redução no M90). Resultado semelhante foi observado por Jalanka (1989b), em cuja pesquisa houve oscilações significativas nesse parâmetro nos momentos 15 e 45 minutos. Todavia, a associação medetomidina-cetamina em leões causou diminuição significativa da frequência respiratória em quatro dos cinco animais anestesiados (Tomizawa et al., 1997), o que demonstra respostas diferentes entre espécies distintas.

A ocorrência de hipóxia não foi uma complicação observada no protocolo testado. Além disso, a maior frequência respiratória no presente estudo, quando comparada à obtida por Lescano et al. (2014) em onças-pardas, pode ser explicada pelo fato de, no presente estudo, não se ter utilizado benzodiazepínico. $\mathrm{O}$ zolazepam causa significativo relaxamento muscular, o que pode ser responsável pela maior depressão respiratória (Williams et al., 2002; Tranquilli et al., 2013).

A medetomidina induz sedação e analgesia dosedependente em relação tanto à duração como à intensidade (Jalanka e Roeken, 1990). Desse modo, foi verificado que existe grande variação na duração dos efeitos da associação medetomidinacetamina em felinos, o que pode ser atribuído às diferentes doses utilizadas entre os estudos. A associação de medetomidina $(80-100 \mu \mathrm{g} / \mathrm{kg}) \mathrm{com}$ cetamina $(7 \mathrm{mg} / \mathrm{kg})$ foi eficiente em propiciar anestesia durante 20-40min em 295 gatos (VahaVahe, 1989). Entretanto, grande parte dos estudos com essa associação farmacológica realiza a reversão da anestesia em 30 a 45 minutos após a indução anestésica (Young e Jones, 1990; Langan et al., 2000; Harrison et al., 2011). A associação de medetomidina $(60 \mu \mathrm{g} / \mathrm{kg})$ com cetamina $(10 \mathrm{mg} / \mathrm{kg})$, em felinos, levou à anestesia de duração de 128,5min (95,1-142,8min), sendo esse tempo considerado longo pelos autores (Zeiler et al., 2014). No presente estudo, a recuperação dos 
animais ocorreu apenas após a administração do reversor.

Longos períodos de anestesia estão de acordo com os achados em leopardo-das-neves (Jalanka, 1989a,b), em cuja pesquisa a associação anestésica de $60-80 \mu \mathrm{g} / \mathrm{kg}$ de medetomidina com $2-3 \mathrm{mg} / \mathrm{kg}$ de cetamina resultou na imobilização por $1,5 \mathrm{~h}$, momento no qual os animais começam a piscar e demonstrar alguma movimentação nas orelhas. Entretanto, os animais permaneceram deprimidos por até nove horas, até completa recuperação. Os autores destacam que, no grupo em que foi aplicado atipamezole, houve diminuição significativa do tempo de recuperação, com os animais em estação após 13,1 $\pm 6,7$ min da aplicação intramuscular. Assim, nas imobilizações realizadas com a associação medetomidina-cetamina em grandes felinos, apesar de vários procedimentos durarem mais de 60min, a reversão se faz necessária (Jalanka e Roeken, 1990).

A associação de medetomidina $(67 \pm 16,1 \mu \mathrm{g} / \mathrm{kg})$ e cetamina $(2,7 \pm 0,8 \mathrm{mg} / \mathrm{kg})$, em leões, propiciou tempo médio de anestesia de $57 \mathrm{~min}$, momento no qual foi aplicado atipamezole (Tomizawa et al., 1997). Os autores destacaram, entretanto, que, no momento em que o reversor foi aplicado, todos os animais ainda permaneciam completamente imobilizados, afirmando-se, assim, que a duração da contenção química gerada pela associação anestésica duraria muito mais do que uma hora.

Uma das principais vantagens da utilização de agonista $\alpha-2$ adrenérgico nas associações farmacológicas para contenção química é a existência de antagonistas, que permitem reversão rápida e suave dos efeitos depressores dos sedativos (Langan et al., 2000; Harrison et al., 2011). No presente estudo, o tempo para decúbito esternal após aplicação do reversor foi significativamente menor nos animais do grupo atipamezole, não havendo diferença estatística, entretanto, no tempo de recuperação entre os dois grupos analisados. Em se tratando de animais silvestres, a rápida reversão dos efeitos anestésicos tem especial importância, uma vez que, além de reduzir os riscos da anestesia, permite liberar o animal mais rapidamente, proporcionando um retorno anestésico menos estressante. Nesse sentido, o uso de protocolos anestésicos que permitem a reversão dos efeitos deve ser preferido para essas espécies, havendo, no presente estudo, aplicação prática equivalente entre os dois reversores.

Sontakke et al. (2009) avaliaram a utilização de ioimbina $(0,1-0,15 \mathrm{mg} / \mathrm{kg})$ intravenosa na recuperação anestésica de felídeos selvagens anestesiados com xilazina associada à cetamina $\mathrm{e}$ observaram que a aplicação do reversor promoveu recuperação anestésica rápida e suave, havendo recuperação completa dentro de $10 \mathrm{~min}$. Todavia, no presente estudo, obteve-se recuperação completa apenas após $92 \pm 24 \mathrm{~min}$, podendo essa diferença ser atribuída às diferentes vias de aplicação. Já Lescano et al. (2014) obtiveram recuperação em 83,3 $\pm 35 \mathrm{~min}$ (após aplicação intramuscular de ioimbina $0,125 \mathrm{mg} / \mathrm{kg}$ ), tempo esse semelhante ao obtido neste estudo, apesar das diferentes doses utilizadas.

Ao analisarem o tempo de reversão anestésica com atipamezole, Langan et al. (2000) obtiveram recuperação rápida $(4,1 \pm 3 \mathrm{~min})$, sendo a dose total $(236,8 \pm 51,2 \mu \mathrm{g} / \mathrm{kg})$ dividida para aplicação pelas vias intravenosa e subcutânea. $O$ tempo de recuperação foi menor ao aqui obtido, o que pode ser atribuído às diferentes vias de aplicação do reversor. Já Miller et al. (2003) utilizaram $129,1 \pm 13,7 \mu \mathrm{g} / \mathrm{kg}$ por via intramuscular, observando tempo médio de recuperação anestésica $(14,2 \pm 5,8 \mathrm{~min})$ inferior ao obtido no presente estudo $(63,3 \pm 23,4 \mathrm{~min})$, apesar da mesma via de aplicação.

Entretanto, essa diferença pode ser atribuída à diferença metodológica da definição de recuperação anestésica, uma vez que os autores acima citados consideraram como recuperação o momento em que o animal entra em decúbito esternal, sendo esse valor semelhante ao obtido no presente estudo. Após aplicação de atipamezole intramuscular, Tomizawa et al. (1997) verificaram que os primeiros sinais para recuperação se iniciaram entre 1 e 10min, entretanto os animais andaram apenas após $53 \mathrm{~min}$, tempo semelhante ao obtido no presente estudo, no qual a recuperação completa ocorreu após $63 \pm 23 \mathrm{~min}$ da aplicação de atipamezole

Os autores do presente estudo reconhecem que o pequeno número de animais avaliados foi um fator limitante, dificultando a detecção de diferenças significativas nos parâmetros observados e impossibilitando a inclusão de um grupo controle. Além da dificuldade de se 
obterem grandes amostras quando se avaliam animais silvestres, o número amostral se deve ao fato de que as anestesias foram realizadas para auxílio na rotina de manejo clínico dos animais no centro de reabilitação, que, na época, reabilitava 11 onças-pardas.

\section{CONCLUSÃO}

A associação de medetomidina $(100 \mu \mathrm{g} / \mathrm{kg}) \mathrm{com}$ cetamina $(5 \mathrm{mg} / \mathrm{kg})$ promoveu anestesia eficiente, rápida e segura em onças-pardas, permitindo a manipulação após $13 \pm 10 \mathrm{~min}$ da aplicação intramuscular e a avaliação durante os 90 minutos de monitorização. Entretanto, foi necessária a aplicação de bolus de propofol para a possibilidade de intubação orotraqueal. Além disso, não foram observadas complicações anestésicas com a utilização desse protocolo. Apesar de o tempo para decúbito esternal ser significativamente inferior após aplicação de atipamezole (18 \pm 7 minutos), quando comparado ao grupo ioimbina ( $36 \pm 17$ minutos), ambos os reversores analisados levaram estatisticamente o mesmo tempo para recuperação anestésica completa $(63 \pm 23$ minutos do grupo atipamezole e $92 \pm 24$ minutos do grupo ioimbina) após aplicação intramuscular, havendo, assim, aplicação prática equivalente entre esses dois reversores.

\section{REFERÊNCIAS}

ALBUQUERQUE, V.B.; ARAÚJO, M.A.; OLIVEIRA, A.R. et al. Evaluation of cardiopulmonary parameters and recovery from anesthesia in cougars (Puma concolor) anesthetized with detomidine/ketamine and isoflurane or sevoflurane. Pesqui. Vet. Bras., v.36, p.33-38, 2016.

ALVES, T.; BRAZ, J.R.C.; VIANNA, P.T.G. A2agonistas em anestesiologia: aspectos clínicos e farmacológicos. Rev. Bras. Anestesiol., v.50, p.396-404, 2000

CANFRÁN, S.; BUSTAMANTE R.; GONZÁLEZ, P. et al. Comparison of sedation scores and propofol induction doses in dogs after intramuscular administration of dexmedetomidine alone or in combination with methadone, midazolam, or methadone plus midazolam. Vet. J., v.210, p.56-60, 2016
CARDOSO, C.G.; MARQUES, D.R.; SILVA, T.H.; MATTOS-JUNIOR, E. Cardiorespiratory, sedative and antinociceptive effects of dexmedetomidine alone or in combination with methadone, morphine or tramadol in dogs. Vet. Anesth. Analg., v.41, p.636-643, 2014

GOODRICH, J.M.; KERLEY, L.L.; SCHLEYER, B.O. et al. Capture and chemical anesthesia of Amur (Siberian) tigers. Wildl. Soc. Bull., v.29, p.533-542, 2001.

HAAPALINNA, A.T.; VIITAMAA, E.; MACDONALD. Evaluation of the effects of a specific alpha 2-adrenoceptor antagonist, atipamezole, on alpha 1- and alpha 2adrenoceptor subtype binding, brain neurochemistry and behaviour in comparison with yohimbine. Naunyn-Schmiedeberg's Arch. Pharmacol. v.356, p.570-582, 1997

HARRISON, K.A.; ROBERTSON, S.A.; LEVY, J.K., ISAZA, N.M. Evaluation of medetomidine, ketamine and buprenorphine for neutering feral cats. J. Feline Med. Surg., v.13, p.896-902, 2011

JACQUIER, M.; AARHAUG, P.; ARNEMO, J.M.; BAUER, H.; ENRIQUEZ, B. Reversible immobilization of free-ranging African lions (Panthera leo) with medetomidine-tiletaminezolazepam and atipamezole Journal Wildl. Dis., v.42, p.432-436, 2006.

JALANKA, H.H. Evaluation and comparison of two ketamine-based immobilization techniques in snow leopards (Panthera uncia). J. Zoo Wildl. Med., v.20, p.163-169, 1989b.

JALANKA, H.H. Medetomidine-and ketamineinduced immobilization of snow leopards (Panthera uncia): doses, evaluation, and reversal by atipamezole. J. Zoo Wildl. Med., v.20, p.154162, 1989a.

JALANKA, H.H.; ROEKEN, B.O. The use of medetomidine, medetomidine-ketamine combinations, and atipamezole in nondomestic mammals: a review. J. Zoo Wildl. Med., v.85, p.259-282, 1990.

JOHANSSON, Ö.; MALMSTEN, J.; MISHRA, C. et al. Reversible immobilization of freeranging snow leopards (panthera uncia) with a combination of medetomidine and tiletaminezolazepam. J. Wildl. Dis., v.49, p.338-346, 2013. 


\section{Caramalac et al.}

LANGAN, J.N.; SCHUMACHER, J.; POLLOCK, C. et al. Cardiopulmonary and anesthetic effects of medetomidine-ketaminebutorphanol and antagonism with atipamezole in servals (Felis serval) J. Zoo Wildl. Med., v.31, p.329-334, 2000

LESCANO, J.; QUEVEDO, M.; BASELLY, L.; CRESPO, A.; FERNÁNDEZ, V. Chemical immobilization of captive Cougars Puma concolor (Linnaeus, 1771)(Carnivora: Felidae) using a combination of tiletamine-zolazepam, ketamine and xylazine. J. Threatened Taxa, v.6, p.6659-6667, 2014.

MILLER, M.; WEBER, M.; NEIFFER, D. et al. Anesthetic induction of captive tigers (Panthera tigris) using a medetomidine-ketamine combination. J. Zoo Wildl. Med., v.34, p.307-308. 2003.

PORTERS, N.; BOSMANS, T.; DEBILLE, M. et al. Sedative and antinociceptive effects of dexmedetomidine and buprenorphine after oral transmucosal or intramuscular administration in cats. Vet. Anesth. Analg., v.41, p.90-96, 2014.

RASZPLEWICZ, J.; MACFARLANE, P.; WEST, E. Comparasion of sedation scores and propofol induction doses in dogs after intramuscular premedication with butorphanol and either dexmedetomidine or medetomidine. Vet. Anaesth. Analg., v.40, p.584-589, 2013.

ROCKHILL, A.P.; CHINNADURAI, S.K.; POWELL, R.A.; DEPERNO, C.S. A comparison of two field chemical immobilization techniques for Bobcats (Lynx rufus). J. Zoo Wildl. Med., v.42, p.580-585, 2011

SINCLAIR, M.D. A review of the physiological effects of $\alpha 2$-agonists related to the clinical use of medetomidine in small animal practice. Can. Vet. J., v.44, p.885, 2003.

SONTAKKE, S.D.; UMAPATHY, G.; SHIVAJI, S. Yohimbine antagonizes the anaesthetic effects of ketamine-xylazine in captive Indian wild felids. Vet. Anaesth. Analg., v.36, p.34-41, 2009
TOMIZAWA, N.; TSUJIMOTO, T.; ITOH, K. et al. Chemical restraint of African lions (Panthera leo) with medetomidine-ketamine. J. Vet. Med. Sci., v.59, p.307-310, 1997.

TRANQUILLI, W.J.; THURMON, J.C.; GRIMM, K.A. (EdS.). Lumb and Jones' veterinary anesthesia and analgesia. New York: John Wiley \& Sons, 2013.

VÄHÄ-VAHE, T. Clinical evaluation of medetomidine, a novel sedative and analgesic drug for dogs and cats. Acta Vet. Scand., v.30, p.267-273, 1989.

VIRTANEN, R.; SAVOLA, J.M.; SAANO, V.; NYMAN, L. Characterization of the selectivity, specificity and potency of medetomidine as an $\alpha 2-$ adrenoceptor agonist. Eur. J. Pharmacol., v.150. p.1-2,9-14, 1988

WILLIAMS, L.S.; LEVY, J.K.; ROBERTSON, SA. et al. Use of the anesthetic combination of tiletamine, zolazepam, ketamine, and xylazine for neutering feral cats. J. Am. Vet. Med. Assoc., v.220, p.1491-1495, 2002.

YAMADA, T.; Idiopathic ventricular arrhythmias: relevance to the anatomy, diagnosis and treatment. J. Cardiol., v.68, p.463-471, 2016.

YOUNG, L.E.; JONES, R.S. Clinical observations on medetomidine/ketamine anaesthesia and its antagonism by atipamezole in the cat. J. Small Anim. Pract., v.31, p.221-224, 1990.

ZEILER, G.E.; DZIKITI, B.T.; FOSGATE, G.T. et al. Anaesthetic, analgesic and cardiorespiratory effects of intramuscular medetomidine-ketamine combination alone or with morphine or tramadol for orchiectomy in cats. Vet. Anaesth. Analg., v.41, p.411-420, 2014. 